\title{
3D electron diffraction on nanoparticles with a complex structure.
}

\author{
M. Gemmi ${ }^{1}$, E. Mugnaioli ${ }^{1}$, R. Kaiukov², S. Toso², L. De Trizio², L. Manna ${ }^{2}$ \\ ${ }^{1}$ Center for Nanotechnology Innovation@NEST, Istituto Italiano di Tecnologia, Piazza S. Silvestro 12, 56126 Pisa, Italy ${ }^{2}$ Department \\ of Nanochemistry, Istituto Italiano di Tecnologia, Via Morego 30, 16163 Genova, Italy
}

mauro.gemmi@iit.it

The synthesis of inorganic compounds in form of nanoparticles of few nanometers has opened a new world for chemistry, with the discovery of unexpected properties and also of entirely new crystal structures. At the beginning, simple stoichiometries related with crystal structures of low complexity have been mainly explored. As far as nanochemistry grew searching for exotic properties, the exploration has extended towards more complex phase diagrams, where the complexity of the crystal structure is a real challenge. In these cases, the crystallographer is hampered by the limited crystal size that enlarges the powder x-ray diffraction peaks and quite often cannot rely on the knowledge of the bulk structure, which can be different from the nanocrystalline form or even not stable in the same conditions. Conversely, 3D electron diffraction (3D ED) has demonstrated its potential for solving crystallographic problems where the size of the crystal grains was the limiting factor [1]. A 3D ED single crystal diffraction experiment is performed with a beam that can be as small as few hundreds of nanometers, and the collected 3D intensity data sets are suitable for structure solution [2]. We report here the application of 3D ED to extreme cases, where the size of the crystalline grains was smaller or in the range of $100 \mathrm{~nm}$ and the powder $\mathrm{x}$-ray diffraction was not able to give a definite answer. The challenge is to establish which is the minimum crystal size that we can investigate in this way. All the nanoparticles we analysed have unknown and not trivial crystal structures.

As first example we report the crystal structure of $\mathrm{Cu}_{2-\mathrm{x}} \mathrm{Te}$, a not stoichiometric plasmonic nanocrystal that exhibits a complex $1 \times 3 \times 4$ super-structure of a pseudo-cubic basic cell, due to the ordering of copper vacancies. The pseudosymmetry of the underlying basic structure induces a strong twinning and therefore data on single individuals could be taken only from grains smaller than 150nm. 3D ED allowed the determination of the super-structure with the identification of $27 \mathrm{Te}$ and $32 \mathrm{Cu}$ in the asymmetric unit and the location of copper vacancies [3].

A second example is the perovskite-related structure of $\mathrm{Cs}_{3} \mathrm{Cu}_{4} \mathrm{In}_{2} \mathrm{Cl}_{13}$ nanocrystals. This crystal structure was synthesized with the aim to obtain a double perovskite of composition $\mathrm{Cs}_{2} \mathrm{CuInCl}_{6}$, isostructural to $\mathrm{Cs}_{2} \mathrm{AgInCl}_{6}$. 3D ED on nanoparticles of $100 \mathrm{~nm}$ revealed that the obtained structure is instead a vacancy ordered perovskite, $\mathrm{A}_{2} \mathrm{BX}_{6}$, in which $25 \%$ of the $\mathrm{A}$ sites are occupied by $\left[\mathrm{Cu}_{4} \mathrm{Cl}\right]^{3+}$ clusters and the remaining $75 \%$ by $\mathrm{Cs}^{+}$, while the $\mathrm{B}$ sites are occupied by $\mathrm{In}^{3+}$ ions. Interestingly, while a Rietveld refinement on powder $\mathrm{x}$-ray data results in a crystal structure where $\mathrm{Cs}^{+}$and $\left[\mathrm{Cu}_{4} \mathrm{Cl}\right]^{3+}$ are disordered on 8 equivalent sites, 3D ED shows that they exists nanoparticles where $\left[\mathrm{Cu}_{4} \mathrm{Cl}\right]^{3+}$ clusters and $\mathrm{Cs}^{+}$are ordered on different sites, lowering the symmetry from cubic Fm-3m to cubic $P n-3 m$ [4].

The last example is the crystal structure determination of $\mathrm{Pb}_{4} \mathrm{~S}_{3} \mathrm{Br}_{2}$, a compound never reported in bulk that we synthesised in form of nanoparticles. 3D ED revealed that this compound is isostructural with the high pressure phase of $\mathrm{Pb}_{4} \mathrm{~S}_{3} \mathrm{I}_{2}$ and attested that the colloidal synthesis is able to freeze a high pressure metastable phase in form of nanoparticles. 3D also revealed that once the size of the nanoparticles has increased above a certain size $(>50 \mathrm{~nm})$ and their shape has changed from spherical to elongated platelets, the structure relaxes with the longest cell parameter that increase from 14.6 to $15.5 \AA$ [5]. In this last case we have reached our minimum crystal size, being able to reconstruct the 3D reciprocal space of a $50 \mathrm{~nm}$ nanoparticle. The examples reported demonstrate that 3D ED is a powerful tool for exploring the crystal structure of not trivial nanoparticles and we expect that, with the use of smaller parallel beam and a dedicated set up, this limit can be pushed further to investigate the crystal structure of nanoparticles in the $10 \mathrm{~nm}$ range.

[1] Gemmi, M., Mugnaioli, E., Gorelik, T.E., Kolb, U., Palatinus, L., Boullay, P., Hovmöller, S., Abrahams, J.P. (2019). ACS Cent. Sci. 5, 1315.

[2] Gemmi, M., Lanza, A. (2019). Acta Crystallogr. B75, 495.

[3] Muganioli, E., Gemmi, M., Tu, R., David, J., Bertoni, G., Gaspari, R., De Trizio, L., Manna, L. (2018). Inorg. Chem. $57,10241$.

[4] Kaiukov, R.,Almeida, G., Marras, S., Dang, D., Baranov, D., Petralanda, U., Infante, I., Mugnaioli, E., Griesi, A., De Trizio, L., Gemmi, M., Manna, L. (2020). Inorg. Chem. 59, 548.

[5] Toso, S., Akkerman, Q. A., Martín-García, B., Prato, M., Zito, J., Infante, I., Dang, Z., Moliterni, A., Giannini, C., Bladt, E., Lobato, I., Ramade, J., Bals, S., Buha, J., Spirito, D., Mugnaioli, E., Gemmi, M., Manna L. (2020). J. Am. Chem. Soc. 142, 10198.

\section{Keywords: electron diffraction, nanochemistry}

Acta Cryst. (2021), A77, C78 